\title{
IMAGE RETOUCHING AND IT'S DETECTION - A SURVEY
}

\author{
Meenakshi Sundaram.A ${ }^{1}$, Nandini. $C^{2}$ \\ ${ }^{1}$ Research Scholar, VTU, Belgaum \\ ${ }^{2}$ Department of Computer Science \& Engineering, Dayananda Sagar Academy of Technology and Management, \\ Bangalore.
}

\begin{abstract}
Image retouching is one more type of image forgery tool which is most commonly used for commercial and aesthetic applications. Retouching operation is carried out mostly to enhance or reduce the image features. Retouching is also done to create a convincing composite of two images which may require rotation, resizing or stretching of one of the image. The paper discusses the state of the art studies organized towards various image authentication techniques and algorithms in digital image Retouching process and its detection. The study also highlights a survey and comparative analysis of various image Retouching mechanisms and some detection methods.
\end{abstract}

Keywords - Digital Image Processing, Image Retouching, image Inpainting, Image Forensics

\section{INTRODUCTION}

Image retouching is one more type of image forgery tool which is most commonly used for commercial and aesthetic applications. Retouching operation is carried out mostly to enhance or reduce the image features. Retouching is also done to create a convincing composite of two images which may require rotation, resizing or stretching of one of the image. Retouching is the process of removing or inserting some given targets into a real photo or image to deliberately to produce an another image. It finds its use in many applications like image coding, journalism, art, film special effects production, virtual reality simulation etc.. Artists professionally retouch the images to restore old historical paintings, Malicious users may also use these manipulations to Tamper the digital images.
Image retouching is also called Image In painting and is used in enhancing or reducing image features and it does not conform to the standards of morality.. Image retouching detection is carried out by trying to find the blurring, enhancements, color changes and illumination changes in the forged image. Detection is easy if the original image is available however blind detection is a challenging task. For this type of forgery two type of modification is done either global or local. Local modification is done usually in copymove and in splicing forgery. Contrast enhancement that is carried out in case of retouching is done at global level and for detection of tampering these are investigated. For illumination and changes in contrast global modification is carried out.
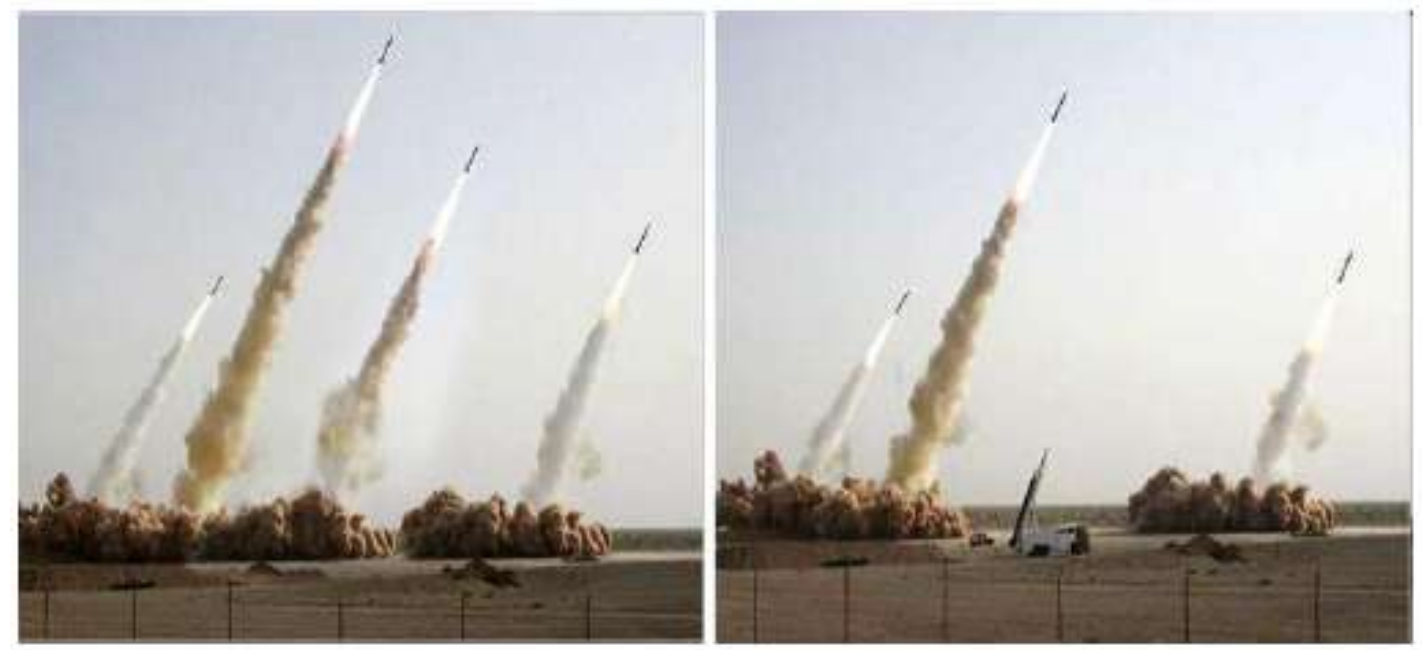

Fig 1 : An example of Image retouching

Example is shown below in Fig1; this photograph was released by Iran army to exaggerate their army strength by simply showing four missiles in place of three in the original image
Image Retouching can be considered as a less harmful kind of digital image forgery [1]. Retouching is nothing but applying some image processing algorithms to enhance or to reduce certain features of an image. But it doesn't 
meaningfully change the image. It can be considered as soft forging. This type of forging is mainly used by magazine photo editors to make the picture more attractive, still meaningful that this kind of image modification is morally wrong.

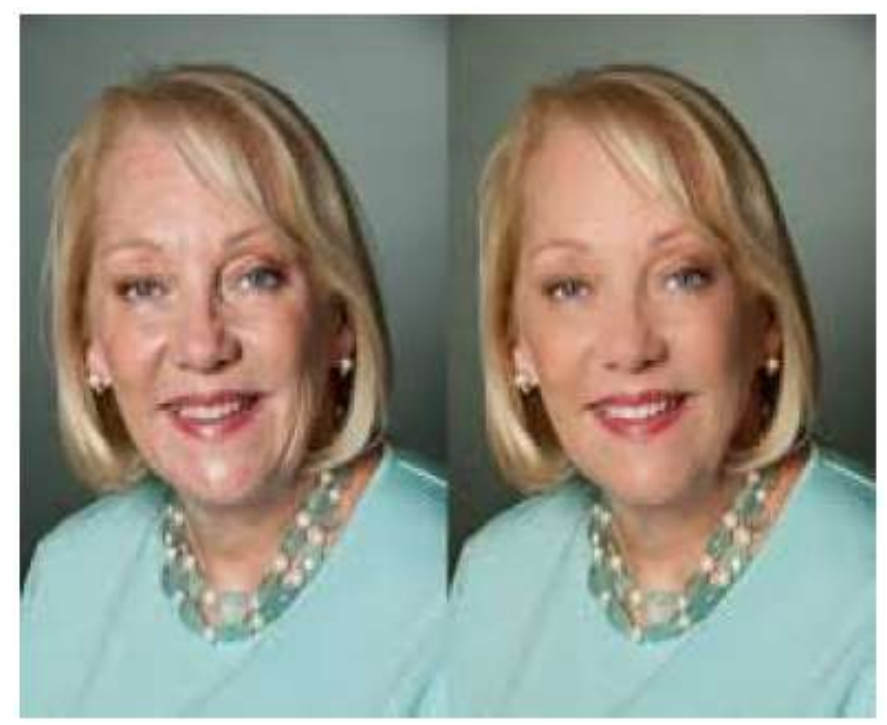

Fig.2: Example of Image Retouching

In IEEE Digital library, the keyword "Image Retouching" gives only 5 conferences and 1 journal/magazine as a search result, which shows that the image retouching has been less studied by many academician and researchers.

\section{EXISTING TECHNIQUES}

Digital image forgery detection methods (DIFDT) are categorized into active and passive approaches [2]. In the active approach, the digital image requires some preprocessing such as watermark embedding or signature generation at the time of image acquisition; it is mainly based on the watermark computation by the camera. Cryptographic digital signatures and Digital watermarking are being used as a means of image authentication in many applications. The downside of this practice is that a watermark must be interleaved at the time of capturing; it limits this approach to specially equipped digital cameras. Moreover, there are millions of digital images already on the internet without watermark or digital signature. Hence, in such situation active approach are not feasible to find the authenticity of the image. In contrast to active approaches, passive techniques operate without any requirement of watermarks or signature embedded in advance. These techniques can be applicable in the concept of notion where digital fakes may escape no visual clues that indicate tampering, they may change the fundamental statistics of the image.

Passive techniques assume that original image patterns and statistics are different even after some image processing. Since the original patterns are constrained by the statistical data..Passive techniques for image forensics operate in the absence of any watermark or signature. These techniques work on the assumption that although digital forgeries may leave no visual clues that indicate tampering, they may alter the underlying statistics of an image. One of the active methods, image forging is mainly categorized into three types, namely Image retouching, Image splicing and CopyMove attack.

The basic algorithms used in image in painting are

a) Linear Interpolation.

b) Texture synthesis which looks for a matching neighborhood and uses it to repair the damaged part.

c) Edge in-ward Expansion used to expand along the damaged edge to inside of the image

d) Radial basis function which chooses a radial basis function as the interpolation function

\section{RELATED WORK}

The survey was conducted by Ruzic et al. [3] a novel MRFdepends in painting technique that employs contextaware approach to decrease the number of potential labels per MRF node.

In the paper demonstrated by $\mathrm{Xu}$ et al. [4] ,a new algorithm which depends on 8-neighborhood rapid sweeping technique is presented. Experimental result demonstrates that there is substantive amplify in the rate of image in painting while remaining good quality effect.

In the review presented by Wang et al. [5] a universal construction for image where three crucial factors are presented such as Area, Shape, and Perimeter (ASP). Then a Pyramid model depend Down-sampling in painting (PDI) model has been presented which is based according to the ASP principles. Experimental outcomes highlights that the performances of existing techniques may be extremely modified after including the PDI model.

In the survey proposed by Huang et al. [6] , an algorithm to attain the greatest patch in every filling step for the target region is proposed. To resolve the challenging problem of repairing organization in image completion, the authors have designed a novel image conclusion approach that depends on mechanical salient organization propagation.

In The paper authored by Bo et al. [7] , image in painting technique that depends on self-organizing map (SOM) is utilized to find the useful structure information of the spoiled image.

The review was illustrated by Gomathi et al. [8] on a new sparse representation-Discrete Shear let Transform is extremely spirited in image in painting submissions together in terms of presentation and computational competence.

Lin et al. [9] demonstrated a new image in painting method where paying multi scale salient arrangement propagation is obtainable. The planned method consists of four phases, namely, detection of salient structure(s), in painting of salient structure(s), in painting of surrounding areas of salient structure(s) can be adapted ant colony optimization (ACO), and in painting of remaining absent regions. 
Hosoi et al. [10], in his work a fast and precise image in painting mechanism has been activated and comparative analysis has been done with respect to the subspace. The proposed method delivers a functionality which is defined from more images connected to the entity class in the erudition stage and estimate the absent pixel standards of the input image fitting to the similar object class so as to feat the comparison between the input image and the-subspace in the painting step.

The survey presented by Dhiyanesh et al. [11],summarises the majority successful dissimilarity models in image segmentation.

In the paper that was illustrated by Takahashi et al. [12] a novel image in painting depends on the matrix rank minimization. Presuming that an image may be modeled by the autoregressive (AR) representation, this survey illustrates the image in painting issue as the signal revival problem of an autoregressive (AR) model.

$\mathrm{Gu}$ et al. [13] presented a new in painting approach where it is considered that a reference image exist. Unlike from the solitary spirit in painting, one can obtain the information of the damaged areas from the location image. Epipolar geometry restraint between the two images are included to the exemplarbased image in painting procedure.

The paper was proposed by Guo et al. [14] an exemplardepend in painting algorithm below smoothness constraint. The satisfying order was optimized by establishing a combination energy function, introducing a novel incomplete discrepancy equation restraint and employing new matching principle.

In the work by Sairam et al. [15] a unified framework of geometry and exemplar depend reflection in painting is proposed. A non-local dissimilarity image in painting formulation is introduced employing graph depend regularization.

The review was conducted by $\mathrm{Du}$ et al. [16] of image allowance and computer-graphic. In the log-Fourier sphere, the pictures earlier than and after blurring bears the isometric, therein a move toward of Gaussian shadow kernel recovery depend on Riemannian geodesic is presented, which approximates the blurring-invariant extent between the unique image and blurred one, and convalesce the blur essence from blur representation.

The review was presented by Zarif et al. [17] to be flaxen as greatly as probable by evaluating the four image completion techniques in the similar dataset as well as appliance hardware.

In the paper that was illustrated by Xu et al. [18] observance linear structure, and surface synthesis model has highquality consequence in repairing the surface information, the imperfection image is decaying into structural image and surface image by wavelet transformation, and then the structural image is repaired by partial discrepancy equation model; the texture image is repaired by surface synthesis method.

Table 1: Comparison of different Image Retouching methods

\begin{tabular}{|c|c|c|c|}
\hline Author & Technology & Outcome & Inference \\
\hline $\begin{array}{l}\text { M Shahid Farid } \\
\text { and Hassan chan } \\
\text { (2010) IEEE [19] }\end{array}$ & $\begin{array}{l}\text { Idea of dynamic weighted of different } \\
\text { size weights to fill in the damaged } \\
\text { areas is used. }\end{array}$ & Execution time is less & $\begin{array}{l}\text { Largely damaged areas } \\
\text { are well repaired }\end{array}$ \\
\hline $\begin{array}{l}\text { Abdul R Zubair } \\
(2011) \\
\text { ICAST [20] }\end{array}$ & $\begin{array}{c}\text { Non-interactive automated mechanism } \\
\text { treating as fluid colors that flow or } \\
\text { diffuse }\end{array}$ & $\begin{array}{l}\text { Computational complexity } \\
\text { is less compared to PDE in } \\
\text { painting method }\end{array}$ & $\begin{array}{l}\text { Suitable for interactive } \\
\text { image manipulations }\end{array}$ \\
\hline $\begin{array}{c}\text { Li liwang and } \mathrm{Yi} \\
\text { wan (2012) IEEE } \\
{[21]}\end{array}$ & $\begin{array}{l}\text { In the established image pyramid. } \\
\text { information is repaired low resolution } \\
\text { image is propagated to high resolution } \\
\text { until the original is inpainted }\end{array}$ & $\begin{array}{l}\text { Fast and yields better } \\
\text { conforming with HVS }\end{array}$ & $\begin{array}{l}\text { Computationally three } \\
\text { times faster }\end{array}$ \\
\hline $\begin{array}{l}\text { Shu-chiang, et al } \\
(2013) \\
\text { IEEE [22] }\end{array}$ & $\begin{array}{l}\text { Exemplar-based method with minimum } \\
\text { error boundary of cut technique based } \\
\text { on Particle-swarm optimization }\end{array}$ & $\begin{array}{l}\text { Time used in searching for } \\
\text { similar blocks is reduced }\end{array}$ & $\begin{array}{l}\text { Repairing efficiency is } \\
\text { enhanced }\end{array}$ \\
\hline $\begin{array}{l}\text { Christel et.al } \\
\text { (2013) IEEE [23] }\end{array}$ & $\begin{array}{l}\text { Presents an innovative tool for image } \\
\text { editing based on color harmony theory }\end{array}$ & $\begin{array}{l}\text { A user Interface in C } \\
\text { designed using QT }\end{array}$ & $\begin{array}{l}\text { Choice for user to retouch } \\
\text { di hammonious colors and } \\
\text { optimize harmonious } \\
\text { colrs }\end{array}$ \\
\hline $\begin{array}{l}\text { Zhen Xie.et.al } \\
\text { (2011) IEEE }\end{array}$ & $\begin{array}{l}\text { Proposes an adaptive algorithm for } \\
\text { inpainting based on Block based } \\
\text { technique }\end{array}$ & $\begin{array}{l}\text { Chooses the best fit } \\
\text { algorithm among linear } \\
\text { interpolation texture } \\
\text { synthesis and radial basis } \\
\text { function }\end{array}$ & Time cost is reduced \\
\hline $\begin{array}{l}\text { Liangtian He and } \\
\text { Yilun wiang } \\
\text { (2014) IEEE }\end{array}$ & $\begin{array}{l}\text { Weighted sparse restoration model is } \\
\text { proposed using split Bregman methods } \\
\text { and iterative support detection method } \\
\text { wavelet frame based images }\end{array}$ & $\begin{array}{l}\text { Enhances the sharp edges } \\
\text { significantly }\end{array}$ & $\begin{array}{l}\text { Joint sparsity property of } \\
\text { wavelet frame coefficients } \\
\text { is used }\end{array}$ \\
\hline
\end{tabular}


Table 2: Comparison of different techniques of Detection of Image Retouching.

\begin{tabular}{|c|c|c|c|}
\hline Author & Technology & Outcome & Inference \\
\hline $\begin{array}{l}\text { Zhentong Driet al } \\
\text { (2013) [24] }\end{array}$ & $\begin{array}{l}\text { Guassian blur kemel recovery based on } \\
\text { Riemannain geodesic is tused to } \\
\text { evaluate the blurring invariant quantity } \\
\text { between the blurred one and the } \\
\text { original image }\end{array}$ & $\begin{array}{l}\text { Authenticate retouch image } \\
\text { generated from bluring }\end{array}$ & $\begin{array}{l}\text { Isometry of images before } \\
\text { and after retouching is } \\
\text { exploited. }\end{array}$ \\
\hline $\begin{array}{l}\text { Gang Cao et.al } \\
\text { (2014) IEEE }\end{array}$ & $\begin{array}{c}\text { Histogram peak gap artifacts analysis } \\
\text { is used. }\end{array}$ & $\begin{array}{l}\text { Consistency between } \\
\text { regional artifacts is } \\
\text { checked for detection of } \\
\text { image forgery }\end{array}$ & $\begin{array}{l}\text { Verifies the integrity of } \\
\text { the images }\end{array}$ \\
\hline $\begin{array}{l}\text { Nazre Batool et.al } \\
\text { (2014)IEEE }\end{array}$ & $\begin{array}{l}\text { Detects facial wrinkles /imperfections } \\
\text { by Gabor filter responses tsing } \\
\text { Guassian Mixture model and EM } \\
\text { algorithm. Correction is done by using } \\
\text { Exemplar-based constrained texture } \\
\text { synthesis algorithm }\end{array}$ & $\begin{array}{l}\text { Other skin imperfections } \\
\text { like wound, scar etc are } \\
\text { detected and corrected }\end{array}$ & $\begin{array}{l}\text { Minimum user } \\
\text { intervention needed to } \\
\text { detect and remove facial } \\
\text { wrinkles }\end{array}$ \\
\hline
\end{tabular}

\section{CONCLUSION}

This paper highlights the various essentials methodologies of image forensics and has discussed the various methods of Image retouching and also techniques which can be used for detection of touched part of the image. Finally some of the recently published research literatures were reviewed and open issues along with research gap is discussed. Our work towards future direction will be to develop a architectural model that can effectively overcome such research gap for the purpose of cost effective design of image forensics applications.

\section{REFERENCES}

[1]. P.Deshpande, "Pixel Based Digital Image Forgery Detection Techniques" IJERA, Vol 2, pp.539-543, 2012L.

[2]. Weiqi, QU Zhenhua, P. Feng, H. Jiwu, "A survey of passive technology for digital image forensics." Frontiers of Computer Science in China-Springer, vol.1, Iss.2, pp.166$179,2007$.

[3]. T. Ruzic, and A. Pizurica, "Context-aware patch-based image inpainting using Markov random field modelling", IEEE Transactions on ImageProcessing, Vol. 24, No. 1, 2015

[4]. J. Xu, D. Feng, J. Wu, and Z. Cui, "An image inpainting technique based on 8-neighborhood fast sweeping method", In Communications and Mobile Computing, CMC'09. WRI International Conference, Vol. 3, pp.626-630, 2009.

[5]. M. Wang, B.Yan, and H. Gharavi, "Pyramid model based Downsampling for image in painting", IEEE 17th International Conference on Image Processing, pp. 429-432, 2010

[6]. H-Y. Huang, and C-N.Hsiao, "A patch-based image inpainting based on structure consistence", In Computer Symposium (ICS), International, pp. 165-170, 2010

[7]. C. Bo, Z. Wang, B. Ming, Q.Wang, and S. Zhen, "A structure first image inpainting approach based on selforganizing map (SOM)", In Information Science and Engineering (ICISE), 2nd International Conference, pp. 1-4, 2010

[8]. R. Gomathi, and A. V. A. Kumar, "An efficient GEM model for image inpainting using a new directional sparse representation: Discrete Shear let Transform", In
Computational Intelligence and Computing Research (ICCIC), IEEE International Conference, pp. 1-4, 2010

[9]. C-S. Lin, and J-J.Leou, "Image In painting Using Multi scale Salient Structure Propagation", In Multimedia and Signal Processing (CMSP), International Conference, Vol. 1, pp. 201-204, 2011

[10]. T. Hosoi, K.Kobayashi, K. Ito, and T. Aoki, "Fast image inpainting using similarity of subspace method", In Image Processing (ICIP), 2011 18th IEEE International Conference, pp. 3441-3444, 2011

[11]. B. Dhiyanesh, and K. S. Sathiyapriya, "Image inpainting and image denoising in wavelet domain using fast curve evolution algorithm", In Advanced Communication Control and Computing Technologies (ICACCCT), IEEE International Conference on, pp. 166-169, 2012

[12]. T. Takahashi, K. Konishi, and T. Furukawa, "Rank minimization approach to image inpainting using null space based alternating optimization.", In Image Processing (ICIP), 19th IEEE International Conference on, pp. 17171720, 2012

[13]. C. Gu, G. Jiang, and L. Duan, "An epipolar geometry guided image inpainting method", In Image and Signal Processing (CISP), $5^{\text {th }}$ International Congress on, pp. 347351,2012

[14]. Y. Guo, and L. Li, "Examplar-Based Image Inpainting Under Smoothness Constraint", In Computational and Information Sciences (ICCIS), Fifth International Conference, pp. 163-166, 2013

[15]. V. Sairam, R. R. Sarma, S. Balasubramanian, and A. S. Hareesh, "A unified framework for geometry and exemplar based image inpainting." InImage Information Processing (ICIIP), IEEE Second International Conference, pp. 511515,2013

[16]. Z. Du, X. Li, and Y. Guo, "Exposing Blur Kernel from Retouch Image", In Computer-Aided Design and Computer Graphics (CAD/Graphics), International Conference on, pp. 407-408, 2013

[17]. S. Zarif, I. Faye, and D. Rohaya, "A comparative study of different image completion techniques", In Computer and Information Sciences (ICCOINS), International Conference on, pp. 1-6, 2014

[18]. Y. Xu, and S. Wang, "Image inpainting based on wavelet transformation", In Software Engineering and Service Science (ICSESS), 5th IEEE International Conference, pp. 541-544, 2014 
[19]. Farid, M.S.; Khan, H., "Image inpainting using dynamic weighted kernels," Computer Science and Information Technology (ICCSIT), 2010 3rd IEEE International Conference on , vol.8, no., pp.252,255, 9- 11 July 2010.

[20]. Zubair, A.R., "A non-iterative automated mechanism for image inpainting," Adaptive Science and Technology (ICAST), 2011 3rd IEEE International Conference, vol., no., pp.193,199, 24-26 Nov. 2011

[21]. Lili Wang; Yi Wan, "Multiresolution image inpainting," Computer Science and Automation Engineering (CSAE), 2012 IEEE International Conference on , vol.3, no., pp.22,26, 25-27 May 2012

[22]. Shu-Chiang Chung; Ta-Wen Kuan; Chuan-Pin Lu; Hsin-Yi Lin, "A new approach of image inpainting based on PSO algorithm," Orange Technologies (ICOT), 2013 International Conference on , vol., no., pp.205,209, 12-16 March 2013

[23]. Chamaret, C.; Urban, F., "No-reference HarmonyGuided Quality Assessment," Computer Vision and Pattern Recognition Workshops (CVPRW), 2013 IEEE Conference on , vol., no., pp.961,967, 23-28 June 2013

[24]. Zhenlong Du; Xiaoli Li; Yanwen Guo, "Exposing Blur Kernel from Retouch Image," Computer-Aided Design and Computer Graphics (CAD/Graphics), 2013 International Conference on , vol., no., pp.407,408, 16-18 Nov. 2013 